\title{
Impactos na saúde mental por distanciamento e isolamento sociais pela COVID-19: uma perspectiva brasileira e mundial
}

\author{
Impacts on mental health by Social distancing and isolation by COVID-19: a brazilian and \\ word perspective
}
Impactos en la salud mental por el Distanciamiento y aislamiento social por la COVID-19: una perspectiva brasileña y mundial

\begin{abstract}
Maria Angélica Otero de Melo dos Reis ${ }^{1 *}$, Bruna Castro Correa ${ }^{2}$, Carolina Vitória Batista Moura ${ }^{3}$, Flávia Eduarda Rodrigues Linhares Oliveira ${ }^{4}$, Mariza Silva Nascimento ${ }^{5}$, Raíssa Teixeira Pinto ${ }^{6}$, Sarah Roldão Batista ${ }^{2}$, Vitória Suriani Gomes ${ }^{7}$, Wendy Shayane Oliveira Martins ${ }^{8}$, Gustavo Fonseca Genelhu Soares ${ }^{9}$.
\end{abstract}

\section{RESUMO}

Objetivo: Ampliar a discussão acerca das repercussões e impactos psicológicos provocados pela pandemia de COVID-19 não somente no cenário brasileiro mas também mundial, em decorrência à emergência de saúde vivenciada na atualidade. Revisão bibliográfica: pôde-se observar que o medo da infecção, a frustração e tédio, falta de suprimentos e perdas financeiras, juntamente com outros desencadeadores, causaram manifestações psicológicas importantes como depressão, mau-humor, irritabilidade, perturbação emocional em geral. Por conseguinte, mostra-se a necessidade de intervenção psicológica frente a limitação causada pelo isolamento devido a COVID-19. Reforça-se, ainda, a importância da assistência profissional adequada, de modo a promover sentimentos de conforto, alívio da aflição, acesso à informação correta, auxílio em casos de crise entre outros, que variam conforme a especificidade de cada profissional. Considerações finais: o distanciamento e o isolamento social, por mais que representem práticas que visam preservar a saúde pública, afetam diretamente a saúde mental dos indivíduos, tornando-se necessárias intervenções nesse aspecto.

Palavras-chave: Saúde Mental, COVID-19, Brasil.

\begin{abstract}
Objective: Expand the discussion about the repercussions and psychological impacts caused by the pandemic of COVID-19 not only in the Brazilian scenario but also worldwide, due to the health emergency experienced today. Bibliographic review: it was observed that fear of infection, frustration and boredom, lack of supplies and financial losses, together with other triggers, caused important psychological manifestations such as depression, moodiness, irritability, emotional disturbance in general. Therefore, there is a need for psychological intervention in view of the limitation caused by isolation due to COVID-19. It also reinforces the importance of adequate professional assistance, in order to promote feelings of comfort, relief of distress, access to correct information, assistance in cases of crisis, among others, which vary according to the specificity of each professional. Final considerations: distancing and social isolation, however much they represent practices that aim to preserve public health, directly affect the mental health of individuals, making interventions necessary in this regard.
\end{abstract}

Key words: Mental Health, COVID-19, Brazil.

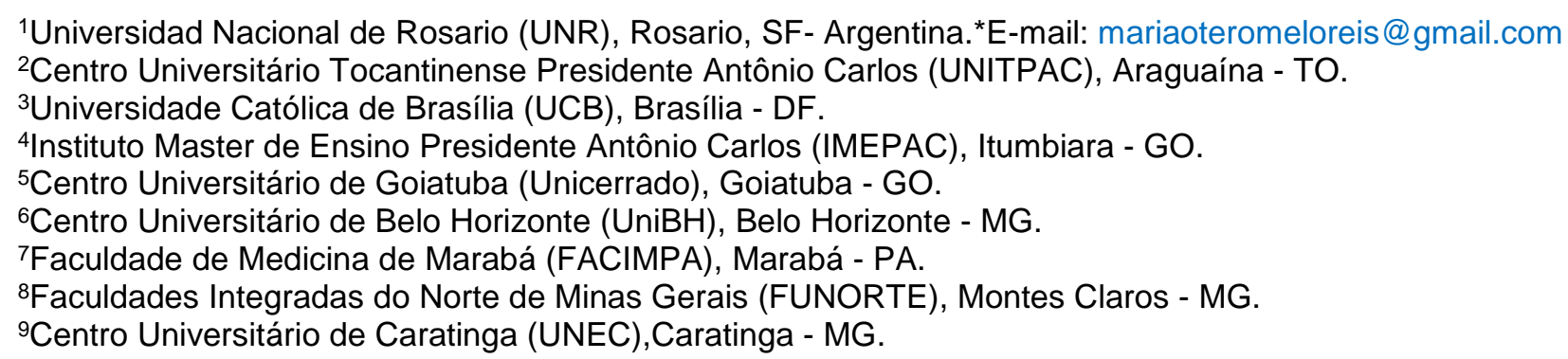




\section{RESUMEN}

Objetivo: Ampliar la discusión sobre las repercusiones e impactos psicológicos provocados por la pandemia de COVID-19 no solo en el escenario brasileño sino también a nivel mundial, debido a la emergencia sanitaria que se vive hoy. Revisión bibliográfica: se observó que el miedo a la infección, la frustración y el aburrimiento, la falta de suministros y las pérdidas económicas, junto con otros desencadenantes, provocaron importantes manifestaciones psicológicas como depresión, mal humor, irritabilidad, trastorno emocional en general. Por lo tanto, existe la necesidad de una intervención psicológica en vista de la limitación provocada por el aislamiento por COVID-19. También refuerza la importancia de una adecuada asistencia profesional, con el fin de promover sentimientos de comodidad, alivio de angustias, acceso a información correcta, asistencia en casos de crisis, entre otros, que varían según la especificidad de cada profesional. Consideraciones finales: el distanciamiento y el aislamiento social, por mucho que representen prácticas que pretenden preservar la salud pública, afectan directamente la salud mental de las personas, por lo que las intervenciones son necesarias en este sentido.

Palabras clave: Salud Mental, COVID-19, Brasil.

\section{INTRODUÇÃO}

O SARS-CoV-2 (Severe Acute Respiratory Syndrome Coronavirus 2), um dos sorotipos de coronavírus responsável pela COVID-19 (do inglês Coronavírus Disease 2019), surgiu na China no final de 2019 e em 2020 espalhou-se pelo mundo, dando origem à pandemia vivenciada na atualidade (RAONY í, et al., 2020). Esta apresenta crescimento exponencial no número de infectados e desencadeia, desde então, sérias repercussões não só nos âmbitos político, econômico e social, mas também biopsicológicos da população (DOS SANTOS MF e RODRIGUES JFS, 2020).

Os sintomas físicos, como febre, cefaléia, mialgia, tosse e ageusia, são bastante comuns e podem ser uma das consequências presentes em indivíduos que adquirem o referido vírus (RAONY í, et al., 2020). No entanto, uma das consequências mais expressivas da pandemia, que muitas vezes é negligenciada, pode ser observada no âmbito psicológico da população (SCHMIDT B, et al., 2020), com impacto na saúde mental causado não só pelo rápido avanço da propagação viral, mas, também, por suas consequências no modo de vida e de interação social dos indivíduos (BEZERRA ACV, et al., 2020; DOS SANTOS MF e RODRIGUES JFS, 2020).

Neste panorama, uma vez que a COVID-19 possui uma alta virulência e poucas evidências de tratamento comprovado cientificamente, a rotina da sociedade entrou em fase de mudança para se adequar à nova realidade (RODRIGUES BB, et al., 2020). Com isso, foram criadas medidas de prevenção que evitassem o contágio do vírus e, assim, grande parte da população e, principalmente, aqueles que se enquadram em grupos de risco, tiveram que cessar suas atividades rotineiras e diárias como o trabalho, o lazer, viagens e estudos (SCHMIDT B, et al., 2020).

Com a intenção de diminuir a propagação do vírus no âmbito nacional, foram necessárias medidas de segurança para impedir a maior propagação da doença infectocontagiosa, dentre elas a quarentena, isolamento ou distanciamento social (BEZERRA ACV, et al., 2020; DOS SANTOS MF e RODRIGUES JFS, 2020). Assim, o Ministério da Saúde (MS), juntamente com a Organização do Mundial da Saúde (OMS), recomendou o distanciamento e isolamento social, acarretando uma mudança drástica na rotina diária dos indivíduos, com impacto nas áreas laboral, pessoal e social, desencadeando fatores importantes, como ansiedade, medo, estresse e pânico na população em geral (SAIDEL MGB, et al., 2020; LIMA RC, 2020).

Com essa mudança de rotina, foram geradas expressivas alterações psicológicas, não só em pessoas com condições já pré-existentes, mas também em indivíduos que nunca apresentaram quadros de acometimentos psicológicos anteriores (RAONY Í, et al., 2020). Além disso, com as modificações impostas pelo isolamento social, a população passou a utilizar com mais frequência e intensidade as mídias sociais e os meios de comunicação virtuais o que gerou, juntamente com o crescimento de acessos, aumento na propagação de informações precipitadas ou equivocadas que contribuem para o agravamento das alterações psicológicas citadas (DOS SANTOS MF e RODRIGUES JFS, 2020). 
Outros possíveis motivos desencadeantes de estresse são o desemprego, gerado pelas expressivas repercussões socioeconômicas da pandemia, e a falta de acompanhamento e tratamento psicológico eficientes que, até recentemente, não eram priorizados pelos profissionais e medidas de saúde (MICHAEL $\mathrm{QD}$, et al., 2020). Além disso, as mudanças psicológicas nos cidadãos, causadas por alterações neuroendócrino-imunológicas, podem causar prejuízo à memória, sintomas depressivos, psicoses e até comportamento suicida (RAONY í, et al., 2020).

Logo, essa é uma questão de relevância ímpar a ser abordada na atualidade, visto que certas medidas são necessárias em prol da saúde e bem estar coletivos (SCHMIDT B, et al., 2020; BEZERRA ACV, et al., 2020). Sendo assim, o presente trabalho visa a ampliação da discussão acerca das repercussões e impactos psicológicos provocados pela pandemia de COVID-19, em decorrência à emergência de saúde vivenciada na atualidade.

\section{REVISÃO BIBLIOGRÁFICA}

O aumento exponencial no número de pessoas infectadas com SARS-CoV-2 está levando à saturação dos serviços de saúde em todo o mundo. Com o intuito de diminuir o avanço da COVID-19 e evitar um colapso no sistema de saúde nacional, os estados e municípios brasileiros estão seguindo as orientações da Organização Mundial da Saúde (OMS) e Ministério da Saúde (MS) e orientando que as pessoas permaneçam em casa como forma de distanciamento social. Outras medidas mais rigorosas, e estratégias para diminuir a disseminação da doença, ficam a critério de cada estado e município (DOS SANTOS MF e RODRIGUES JFS, 2020).

A fim de evitar a transmissão e o aumento no número de casos, foi instituído o período de quarentena em diversos lugares do mundo. Essa medida, segundo o Ministério da Saúde, consiste na separação de pessoas sintomáticas e assintomáticas, com investigação laboratorial, gerando um distanciamento social desses indivíduos por 14 dias, período este que é o tempo que o vírus leva para se manifestar no ser humano. No entanto, este prazo pode aumentar de acordo com o resultado dos exames laboratoriais. Normalmente, o isolamento é feito em casa, com as devidas restrições, ou em hospitais, a depender de cada caso. Essa ação é importante visto a necessidade de separar aqueles que foram infectados, ou tiveram qualquer contato com essa doença contagiosa, pois dessa forma há redução do risco de infecção de outras pessoas pelo do SARSCoV-2 (DOS SANTOS MF e RODRIGUES JFS, 2020).

A quarentena pode ser uma medida preventiva necessária durante grandes surtos de doenças infecciosas, porém também pode ser relacionada a efeitos psicológicos negativos como ansiedade, humor deprimido e irritabilidade (RODRIGUES BB, et al., 2020). Além disso, tais intervenções podem ser diretamente associadas ao aparecimento de doenças agudas e sintomas de estresse (GOULARTE JF, et al., 2020; SIM HS e HOW $\mathrm{CH}, 2020)$.

E dentro desta premissa, milhares de pessoas tiveram que mudar sua rotina de forma drástica, causando, com frequência, repercussões psicossociais severas. Nesse contexto, é válido questionar: quais são as repercussões psicossociais que afetam/afetaram os indivíduos durante o isolamento social (DOS SANTOS MF e RODRIGUES JFS, 2020).

Estudos atuais sobre o comportamento da saúde mental durante a pandemia demonstraram que houve uma maior prevalência de sintomas como: perturbação emocional, depressão, estresse, mau-humor, irritabilidade, insônia e sintomas de estresse pós-traumático. Isso ocorre uma vez que os principais contribuintes ao sofrimento emocional generalizado e aumento do risco de doenças psiquiátricas, nesse contexto, são o medo de infecção, frustração e tédio, a falta de suprimentos e as perdas financeiras, somado a outros desencadeadores importantes como a solidão e o luto (DUARTE MQ, et al., 2020; GOULARTE JF, et al., 2020).

Estudos populacionais realizados até o momento sobre implicações na saúde mental diante da pandemia, revelam que os sintomas de depressão podem ir de moderados a severos, com aumento da possibilidade de suicídio em situações extremas (SCHMIDT B et al., 2020). Na Coréia do Sul, casos de suicídio potencialmente 
ligados às implicações psicológicas do coronavírus também foram reportados aos governantes (SCHIMIDT B, et al., 2020). O autoextermínio, no entanto, possui pontos de incentivos além da depressão, como transtorno de humor bipolar e dependência de álcool. Porém, o medo, a solidão, a tensão econômica e as incertezas sobre o futuro podem potencializar ações suicidas, visto que estes podem ser fatores motivacionais para crises depressivas (BARROS MBA, et al., 2020).

Nesse contexto, as pesquisas apontam que os brasileiros estão mais ansiosos atualmente do que no período pré pandêmico (BARROS MBA, et al., 2020). Nos Estados Unidos, um estudo investigou mais de 10 milhões de buscas no Google e avaliou as mudanças nas consultas de pesquisa de saúde mental após medidas de isolamento social. Observou-se que os tópicos relacionados a ansiedade, pensamentos negativos, distúrbios do sono e ideação suicida aumentaram drasticamente (MELO MCA e SOARES DS, 2020). Pesquisas têm discutido sobre os riscos à saúde associados ao isolamento e solidão, e tem-se observado sua equivalência aos efeitos prejudiciais do fumo e da obesidade (COURTIN E e KNAPP M, 2017).

Os humanos são seres sociais, ou seja, possuem a necessidade de se relacionar com outras pessoas. Dessa forma, manter o isolamento por um longo período pode levar a condições de sofrimento psicológico significativo. Concomitantemente, a maior prática de hábitos de vida não saudáveis como o sedentarismo pode levar a fatores de risco cardiometabólicos, que também estão ativamente relacionados à saúde mental (HAO Y et al., 2020; BALANZÁ-MARTINEZ V, et al., 2020).

Em um cenário de pandemia, as consequências psicossociais em resposta ao contexto atual possuem grande variabilidade individual. Dessa forma, sentimentos de medo, ansiedade e tristeza, embora sejam normais em reação a situação de pandemia, podem afetar as pessoas em diferentes níveis de gravidade. Estudos recentes relatam que grupos mais vulneráveis como os idosos, as crianças, pessoas com alguma deficiência ou histórico de problemas de saúde mental ou outra comorbidade, têm consequências mais graves. Simultaneamente a isso, aqueles que vivem em condições precárias, com acesso difícil e limitado a serviços sociais e de saúde, também são caracterizados como indivíduos de maior vulnerabilidade (LEIVA AM, et al., 2020).

Sendo assim, o impacto psicossocial do COVID-19 no Brasil deve ser tratado como um problema de saúde pública (GOULARTE JF, et al., 2020). Ademais, verificou-se nesta revisão que a saúde mental da população mais pobre tem sido mais prejudicada do que as demais (BEZERRA ACV, et al., 2020). Observa-se que nas faixas de menor renda encontra-se um maior número de pessoas que afirmam ter parado de ganhar dinheiro durante a pandemia, 35\% entre os que declararam não ter renda (BEZERRA ACV, et al., 2020).

Evidentemente, pessoas com renda baixa ou nenhuma renda se sentem mais preocupadas, estressadas e ansiosas devido a sua situação financeira. Soma-se a isso as más condições de habitação e de convívio, que propiciam transtornos psíquicos (SCHMIDT B, et al., 2020). Pesquisas ressaltam, ainda, que a parcela com menor renda está praticando menos o isolamento social em relação àquela com maior renda e isso ocorre, principalmente, em função da necessidade de locomoção para o trabalho. Muitas vezes, esses indivíduos utilizam transportes coletivos que têm um alto índice de contaminação, o que gera aumento do medo de contrair a doença e, consequentemente, aumentando a ansiedade (BEZERRA ACV, et al., 2020).

Os desafios impostos pela pandemia, incluindo o medo da doença, o medo de morrer, a reestruturação da rotina doméstica regular e a perda dos meios de subsistência, podem contribuir significativamente para 0 aumento do estresse (MOREIRA DN e COSTA MP, 2020). É esperado também a sensação recorrente de impotência perante os acontecimentos, irritabilidade, angústia, tristeza e insônia e, durante $o$ isolamento, pode-se intensificar os sentimentos de desamparo, tédio e solidão. Como já citado, alguns grupos podem ser mais vulneráveis do que outros aos efeitos psicossociais das pandemias. Por se encontrarem em um período crítico de desenvolvimento, as crianças e adolescentes merecem cuidados especiais para preservar e promover sua saúde mental (DE MIRANDA DM, et al., 2020).

Outro aspecto a ser considerado nesse cenário é a atuação dos profissionais de saúde e sua maior fragilidade quanto às repercussões sociais e emocionais. Estes precisam lidar diariamente com seus sentimentos de impotência, frustração, estresse pela sobrecarga e condições de trabalho, inseguranças sobre 
a doença e seu tratamento, além do medo de se infectar e também o medo de transmitir o vírus a familiares e/ou pacientes. Não obstante, lidam ainda com as perdas de muitos doentes e de entes queridos, no contexto instável característico de uma pandemia (SAIDEL MGB, et al., 2020).

Diferentes estudos, realizados na China e na Alemanha, identificaram agravantes na população de profissionais da saúde em relação ao quadro atual de pandemia. Entre os agravantes do sofrimento psíquico estão: carga de trabalho excessiva, isolamento, discriminação, medo de se infectar e infectar outros, não ter clareza do diagnóstico, já que os sintomas da COVID-19 são considerados inespecíficos e podem estar presentes em muitas outras doenças, cuidar de familiares (incluindo crianças) que estão passando mais tempo em casa e a preocupação com a saúde mental e física. Isso os torna bastante vulneráveis a sofrer exaustão, medo, perturbações do sono e distúrbios emocionais (LAI J, et al., 2019; PETZOLD MG, et al., 2020;TOROUS J, et al., 2020 apud SAIDEL MGB, et al., 2020).

Uma investigação chinesa evidenciou que, entre 1257 profissionais que estavam cuidando diretamente de pacientes com COVID-19, a metade (50\%) apresentavam depressão e mais de $70 \%$ apresentavam sintomas de angústia, além de ansiedade e insônia. Essas queixas foram maiores em enfermeiras, em mulheres e em profissionais que têm contato direto com pacientes infectados (SAIDEL MGB, et al., 2020). Um possível mecanismo relacionado à infecção por SARS-CoV-2 e o comprometimento da saúde mental poderia ser o envolvimento de redes neuroimunes devido aos níveis circulantes aumentados de várias citocinas próinflamatórias (por exemplo, Interleucina-6 e Interferon-gama) que foram encontrados em pacientes com MERS, SARS ou COVID-19 (RAONY Í, et al., 2020).

As citocinas solúveis que chegam ao cérebro, ou as alterações dos níveis locais correspondentes destas, podem influenciar a síntese, liberação e recaptação de vários neurotransmissores, incluindo monoaminas, como dopamina, norepinefrina e serotonina. Mudanças no metabolismo dos neurotransmissores estão envolvidos na fisiopatologia de vários transtornos psiquiátricos, como a depressão. Uma vez que as alterações nos níveis de citocinas podem levar a uma interrupção no metabolismo dos neurotransmissores, desencadeando déficits comportamentais, há uma hipótese de que um possível mecanismo relacionado à infecção por SARS-CoV-2 e os resultados de saúde mental é o envolvimento de redes neuroimunes (RAONY í, et al., 2020).

Os episódios depressivos caracterizam-se pela presença de sensação de tristeza, vazio ou humor irritável, acompanhado de mudanças somáticas e cognitivas que afetam significativamente a capacidade funcional do indivíduo por pelo menos uma semana. Cada vez mais evidências apontam que o coronavírus pode invadir o sistema nervoso central levando a sintomas psiquiátricos. Um estudo feito em todo o Brasil mostrou que, durante o período da pandemia e de distanciamento social analisado, 40,4\% dos brasileiros sentiram-se tristes ou deprimidos muitas vezes ou sempre (BARROS MBA, et al., 2020).

Além disso, verificou-se que, na área educacional, estudantes universitários e docentes têm apresentado maiores índices de depressão. A interrupção das aulas presenciais e, em alguns casos, a total suspensão dos cursos provisoriamente, gerou uma grande mudança de rotina entre os estudantes e suas perspectivas futuras (MAIA BR e DIAS PC, 2020).

$\mathrm{Na}$ China, um estudo realizado entre janeiro e fevereiro de 2020, que incluiu 1.210 entrevistados de 194 cidades, revelou que $53,8 \%$ dos indivíduos avaliados apresentaram impacto psicológico moderado ou grave, sendo que $16,5 \%$ foram detectados com sintomas depressivos (LEI L, et al., 2020 apud BARROS MBA, et al., 2020).

Já a ansiedade pode ser compreendida como um mecanismo que nos ajuda a detectar o perigo e adotar as medidas necessárias para lidar com ele. No entanto, esse recurso adaptativo muitas vezes pode ficar fora de controle, causando sofrimento e prejuízo ao desempenho social e/ou profissional da população. Ansiosos tendem a possuir vários sintomas físicos diferentes, como fobia social, transtornos de estresse e pânico (DAL'BOSCO EB, et al., 2020). Assim, ao se depararem com um vírus capaz de ocasionar pandemia, mudanças de rotina, perdas e incertezas, as pessoas se sentem apavoradas e, consequentemente, ansiosas (DUARTE MQ, et al., 2020). 
Atualmente, possíveis situações desencadeantes das crises de ansiedade são o consumo exacerbado de notícias (verdadeiras e falsas) sobre a pandemia pela população, aumento de desemprego e o medo frente ao aumento diário do número de mortes e infecções ao redor do país. Nesse contexto, durante o período de pandemia e de distanciamento social, $52,6 \%$ das pessoas referiu sentir-se ansioso ou nervoso sempre ou quase sempre (BARROS MBA, et al., 2020). Estudos também apontam que pessoas com anterior sofrimento psíquico são mais vulneráveis no contexto do isolamento social, pois são mais propensas a terem níveis de estresse mais elevados e maior ansiedade, devido à piora do quadro patológico já adquirido e também à dificuldade em dispor de seus tratamentos adequados (BARROS MBA, et al., 2020).

Os estudos acadêmicos desenvolvidos nesse período emergencial de saúde sugerem a necessidade investigativa do tema, visto que as consequências das dificuldades enfrentadas podem propagar-se posteriormente, e de uma forma não conhecida atualmente (MAIA BR e DIAS PC, 2020). Além disso, até metade da população acometida por epidemias podem sofrer danos psicológicos e psiquiátricos, caso não sejam conduzidas da melhor maneira (LIMA RC, 2020). Dessa forma, em um seguinte contexto, será necessária a elaboração de programas fomentadores da discussão da realidade e medidas com o fito de atenuação dos impactos prejudiciais à saúde mental ocorridos neste período (MAIA BR e DIAS PC, 2020).

Apesar do contexto limitante diante do isolamento social, sugere-se a utilização de estratégias que possibilitem a atividade de assistência profissional humana, bem como suporte social, a partir da forma remota de atendimento, viabilizando o tratamento psicológico, conforto, alívio da aflição e auxílio em casos de crise, bem como o suprimento de alimentos e água. Uma outra medida importante é o acesso à informação correta e objetiva sobre a prevenção, tratamento e situação local da pandemia, de forma a diminuir os níveis de estresse, ansiedade e depressão (SCHMIDT B, et al., 2020). As intervenções psicológicas podem ocorrer de diversas formas, dependendo das especificidades de cada indivíduo (SCHMIDT B, et al., 2020).

No caso dos profissionais de saúde, o acesso a essas intervenções auxilia e gera impactos em todo o contexto social, visto sua importância frente aos desafios enfrentados no trabalho, como a necessidade de mediação psicossocial de emergência, promoção de um ambiente da linha de frente benéfico à saúde mental e prevenção de danos psicológicos futuros (TEIXEIRA CFS, et al., 2020). Em suma, é importante ressaltar a necessidade das práticas de isolamento social e quarentena no controle da propagação de doenças infectocontagiosas e também o risco de danos psicológicos que podem ocorrer caso essas medidas não sejam tomadas (DOS SANTOS MF e RODRIGUES JFS, 2020).

Ademais, pesquisas recentes alertam que as respostas à atual pandemia mostram uma ampla variabilidade individual, sendo capaz de desencadear sentimentos de medo, ansiedade ou tristeza, com diferentes graus de gravidade. As respostas descritas neste artigo, embora sejam reações normais a uma catástrofe, pessoas em condições elevadas de vulnerabilidade social devem ser avaliadas particularmente, fazendo-se urgentemente necessário que os governos elaborem planejamentos e respostas multissetoriais mínimas para ajudar no bem-estar psicossocial da população (LEIVA, AM et al. 2020). Sendo assim, a maior crise de saúde pública enfrentada internacionalmente tem a psicologia como uma importante aliada na reparação do impacto causado devido às consequências da pandemia (SCHMIDT B, et al., 2020).

\section{CONSIDERAÇÕES FINAIS}

Como objetivo geral, o presente estudo se propôs a analisar os impactos causados pela pandemia da COVID-19 na saúde mental da população.Compreendeu-se que, as medidas de contenção da doença trouxeram algumas repercussões negativas, principalmente em ambientes psicossociais mais vulneráveis, diante das situações que exigem quarentena e isolamento social. Diante disso, é necessário que medidas de saúde sejam implementadas, a fim de ajudar a manter a boa qualidade de vida e a saúde psicológica dos atingidos. Vale ressaltar que, a prática do isolamento social e da quarentena são essenciais para o controle da propagação dessa doença infectocontagiosa, porém a preocupação com a saúde em geral não deve ser negligenciada. Neste sentido, sugere-se que iniciativas com a finalidade de cuidar do sofrimento psíquico da população atingida sejam criadas, além de elaborar estratégias voltadas às possíveis mudanças comportamentais de cada indivíduo de forma singular. 


\section{REFERÊNCIAS}

1. BALANZÁ-MARTINEZ V, et al. Lifestyle behaviours during the COVID-19 - time to connect. Acta Psychiatr Scand, 2020 141(5):399-400.

2. BARROS MBA, et al. Relato de Tristeza/Depressão, Nervosismo/Ansiedade e Problemas de Sono Na População Adulta Brasileira Durante a Pandemia de COVID-19. Epidemiologia e Serviços de Saúde, 2020;29 (4).

3. BEZERRA ACV, et al. Fatores associados ao comportamento da população durante o isolamento social na pandemia COVID-19.Ciênc. saúde coletiva[online], 2020; 25(1):2411-2421.

4. COURTIN E, KNAPP M. Social Isolation, Loneliness and Health in Old Age: A Scoping Review. Health \& Social Care in the Community, 2015; 25(3): 799-812.

5. DAL'BOSCO EB, et al. Mental health of nursing in coping with COVID-19 at a regional university hospital. Revista brasileira de enfermagem, 2020; 73.

6. DE MIRANDA DM, et al. How is COVID-19 pandemic impacting mental health of children and adolescents? International Journal of Disaster Risk REductions, 2020; 21(2020):101845.

7. DOS SANTOS MF, RODRIGUES JFS. COVID-19 e repercussões psicológicas durante a quarentena e o isolamento social: uma revisão integrativa. Nursing, 2020; 4095-4100.

8. DUARTE MQ, et al . COVID-19 e os impactos na saúde mental: uma amostra do Rio Grande do Sul, Brasil. Ciênc. saúde coletiva,2020. v. 25, n. 9, p. 3401-3411.

9. GOULARTE JF, et al. COVID-19 and mental health in Brazil: Psychiatric symptoms in the general population. Journal of Psychiatric Research, 2020;132 (2021):32-37.

10. HAO Y, et al. Patients with mental health disorders in the COVID-19 epidemic. The Lancet Psychiatry, $2020 ; 7$.

11. LEIVA AM, et al. Dimensión psicosocial de la pandemia: la otra cara del covid-19. Ciencia yenfermería, $2020 ; 26$.

12. LIMA RC, Distanciamento e isolamento sociais pela Covid-19 no Brasil: impactos na saúde mental.Physis: Revista de Saúde Coletiva,2020; 30(2):e300214.

13. MAIA BR, DIAS PC. Ansiedade, depressão e estresse em estudantes universitários: o impacto da COVID-19. Estud. psicol 2020; 37: e200067.

14. MELO MCA, SOARES DS. Impact of social distancing on mental health during the COVID-19 pandemic: An urgent discussion. International Journal of Social Psychiatry, 2020; 66(6):625-626.

15. MOREIRA DN, COSTA MP. The Impact of the Covid-19 Pandemic in the Precipitation of Intimate Partner Violence. International Journal of Law and Psychiatry, 2020; 71: 101606.

16. RAONY Í, et al. Psycho-neuroendocrine-immune interactions in COVID-19: potential potentials in mental health. Frontiers in Immunology, 2020;11:1170, 2020.

17. RODRIGUES BB, et al. Aprendendo com o Imprevisível: Saúde Mental dos Universitários e Educação Médica na Pandemia de Covid-19. Rev. bras. educ. med., 2020; 44(1): e149.

18. SAIDEL MGB, et al. Intervenções em saúde mental para profissionais de saúde frente a pandemia de Coronavírus [Mental health interventions for health professionals in the context of the Coronavirus pandemic][Intervenciones de salud mental para profesionales de la salud ante la pandemia de Coronavírus]. Revista Enfermagem UERJ, 2020; 28: $p 4992$

19. SCHMIDT B, et al. Saúde mental e intervenções psicológicas diante da pandemia do novo coronavírus (COVID-19). Estud. psicol.2020; 37: e200063

20. SIM HS, HOW CH. Mental Health and Psychosocial Support during Healthcare Emergencies - COVID-19 Pandemic. Singapore Medical Journal, 2020; 61 (7): 357-362

21. TEIXEIRA CFS, et al . A saúde dos profissionais de saúde no enfrentamento da pandemia de Covid-19. Ciênc. saúde coletiva, 2020; 25(9): 3465-3474 